\title{
Three-dimensional Computed Tomography Imaging of a Frontal Skull Base Fracture
}

\author{
-Case Report-
}

\author{
Koh YANO, Tatsuya Kuroda, Yusuke TANABE, Akira TAKaO*, \\ and Noboru SAKAI** \\ Departments of Neurosurgery and *Pediatrics, Gifu Municipal Hospital, Gifu; \\ ** Department of Neurosurgery, Gifu University School of Medicine, Gifu
}

\begin{abstract}
A 7-year-old boy presented with recurrent meningitis after head trauma. Coronal computed tomography (CT) revealed prolapse of the intracranial soft tissue into the right ethmoidal sinus, leading to the diagnosis of right frontal skull base fracture. Three-dimensional CT with bone windows provided a lifelike image of the fracture lateral to the right olfactory groove. This image was most useful in the preoperative planning of the repair surgery.
\end{abstract}

Key words: three-dimensional computed tomography, bone windows, skull base fracture

\section{Introduction}

Bacterial meningitis secondary to skull base fracture is not uncommon. ${ }^{6]}$ However, preoperative determination of the exact location of the fracture is often difficult. ${ }^{3-5,9]}$ Three-dimensional computed tomography (3D-CT) can provide lifelike imaging of the skull base, and has been used to better delineate craniofacial trauma. ${ }^{7}$ We describe a case of recurrent meningitis after head trauma, in which 3D-CT clearly visualized the frontal skull base fracture.

\section{Case Report}

On August 31, 1992, a 7-year-old boy was watching his father's friend inflate an automobile tire when suddenly the tire burst and blew him away high above the ground. He fell to the ground on his head. He was transferred to an emergency hospital where CT showed bilateral frontal lobe contusion. He was treated conservatively and discharged without neurological deficits except for a traumatic cataract of his right eye. The affected eye lens was replaced with an artificial one on December 4, 1992.

On January 29,1993 , he presented to a pediatrician in our hospital with symptoms of high temperature, headache, and vomiting. Cerebrospinal fluid

Received January 31, 1997; Accepted August 4, 1997 culture revealed Streptococcus pneumoniae. Cefotiam was administered under a diagnosis of bacterial meningitis, which was curative. The possibility of skull base fracture was not suspected at this episode. On November 10, 1993, more than 14 months after the accident, he developed meningitis again. The attending pediatrician then suspected that the patient might have skull base fracture due to the accident, although neither the boy nor his parents had noticed apparent cerebrospinal liquorrhea at either episode. Neurosurgical consultation was made and frontal skull base fracture was considered to be likely from CT scan, which was taken at the first episode of meningitis and showed bifrontal subdural fluid accumulation (Fig. 1). Coronal CT confirmed the frontal skull base fracture, demonstrating prolapse of the intracranial tissue into the right ethmoidal sinus (Fig. 2). 3D-CT with bone windows was then performed for the preoperative planning of the repair surgery. 3D-CT demonstrated a fracture site located lateral to the right olfactory groove, which itself was collapsed (Fig. 3).

The second episode of meningitis was treated by administration of cefotaxime and ampicillin. The patient then underwent an operation to repair the frontal skull base fracture on December 24, 1993. A bilateral frontal craniotomy was performed with the patient in the supine position. The right frontal lobe was lifted intradurally, exposing the degenerated ol- 


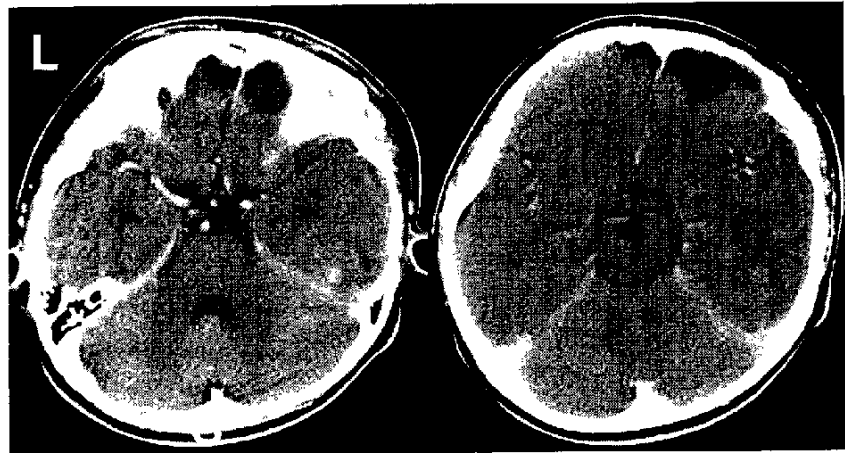

Fig. 1 Axial computed tomography scans demonstrating bifrontal subdural fluid accumulation, greater on the right.
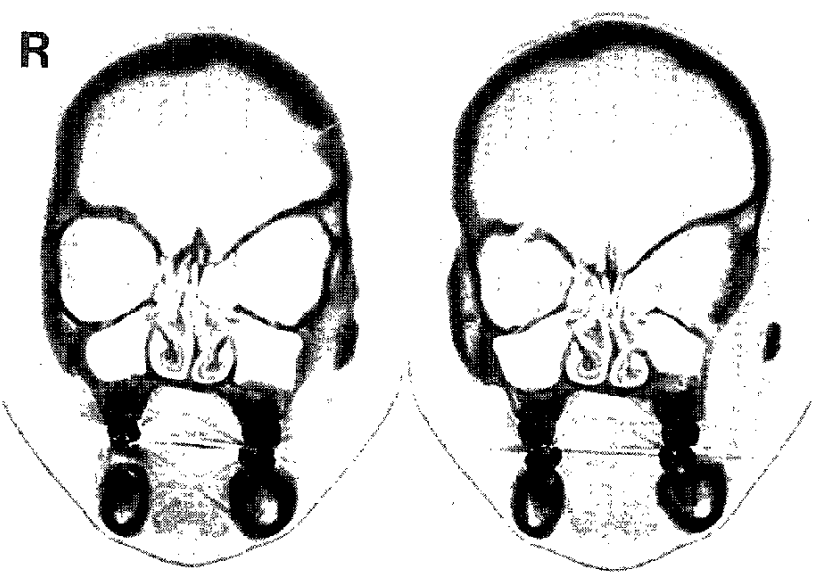

Fig. 2 Coronal computed tomography scans showing prolapse of the intracranial soft tissue into the right ethmoidal sinus.

factory tract on the collapsed olfactory groove. The fracture lateral to the groove was a collection of holes, as shown by 3D-GT. The dura was linearly lacerated along the holes. This intraoperative finding suggested that although the original underlying fracture was linear, intracranial pressure had caused the weakened bones to form holes during a relatively short time after the trauma. The brain prolapsed into the holes was removed and the mucosa of the ethmoidal sinus was pushed down. Pieces of the temporal muscle were used to cap the holes, which were then sealed with fascia reinforced by bioactive adhesives.

He has now been followed up for more than 3 years with no further signs of meningitis.

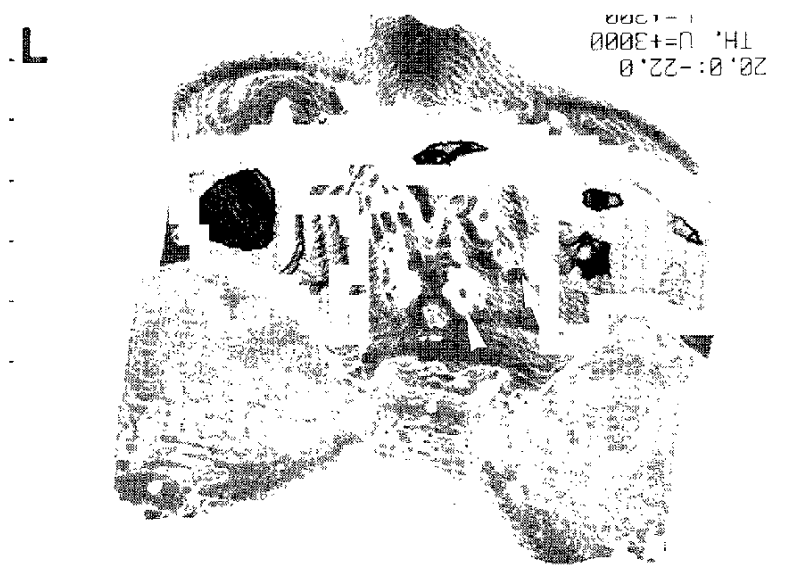

Fig. 3 Three-dimensional computed tomography scan with bone windows demonstrating the fracture lateral to the right olfactory groove. The fracture (arrow) appears as a collection of holes. The right olfactory groove (arrowhead) is collapsed and narrower compared with that on the left.

\section{Discussion}

Cerebrospinal liquorrhea is a well-known indicator of skull base fracture after head trauma. ${ }^{3,6]}$ Conversely, the diagnosis of skull base fracture in cases without cerebrospinal liquorrhea is often difficult, ${ }^{6]}$ especially when the patients are children who are prone to develop bacterial meningitis secondary to other infectious diseases. Unfortunately, the present case was such an occurrence, and the possibility of skull base fracture was overlooked at the first episode of meningitis.

A variety of problems with the diagnostic tools for skull base fracture add to the difficulty in diagnosis. These include: 1) Coronal CT causes patient discomfort due to awkward positioning, and is especially problematic in the pediatric patient who is not cooperative; 2) Radionuclide cisternography needs a cumbersome procedure and may not be useful in cases with no apparent liquorrhea such as ours $^{3)}$; 3) Metrizamide CT cisternography may be sensitive to intermittent cerebrospinal liquorrhea, but requires an invasive procedure. ${ }^{3)}$ From these standpoints, 3D-CT with its simple procedure may be the first candidate for the diagnostic procedure for skull base fracture. However, 3D-CT has its own problem that is high radiation risk. In our patient, 3D-CT was reconstructed from two-dimensional spiral CT images. Spiral CT generates lower dose of radiation per section than conventional $\mathrm{CT}{ }^{2)}$ Therefore, although 3D-CT reconstruction of the skull 
requires data from thinner sections than conventional CT, radiation dose will not be markedly increased if the block of the skull to be reconstructed is limited. ${ }^{1,8)}$

Coronal CT of our patient showed brain prolapse into the right ethmoidal sinus. Although this information was useful for the diagnosis, the lifelike image of the fracture site obtained with 3D-CT was far superior for the surgical planning of the repair. This feature of 3D-CT in preoperative planning has already been advocated. ${ }^{7)}$

We conclude that 3D-CT may be a powerful tool for the diagnosis of skull base fracture, although the diagnostic tool should be carefully tailored to each individual. It should be kept in mind that the merits of 3D-CT such as comfortable positioning, no physical pain, and thus easy cooperation on the part of patients must be weighed against the demerit of it, which is radiation risk of the patient, especially in the pediatric population. Performing repeated $\mathrm{CT}$ scanning in vain should be absolutely avoided.

\section{References}

1) Ali QM, Dietrich B, Becker H: Patterns of skull base fracture: a three-dimensional computed tomographic study. Neuroradiology 36: 622-624, 1994

2) Craven CM, Naik KS, Blanshard KS, Batchelor AGG, Spencer JA: Multispiral three-dimensional computed tomography in the investigation of craniosynostosis: technique optimization. Br J Radiol 68: 724-730, 1995

3) Fagerlund M, Liliequist B: Intermittent cerebrospinal liquorrhea. Cerebral computed tomography in the non-drop period. Acta Radiol 28: 189-192, 1987

4) Laine FJ, Kuta AJ: Imaging the sphenoid bone and basiocciput: Pathological considerations. Semin Ultrasound CT MR 14: 160-177, 1993

5) Laine FJ, Nadel L, Braun IF: CT and MRI imaging of the central skull base: Part 2. Pathological spectrum. Radiographics 10: 797-821, 1990

6) Lui TN, Lee ST: Late posttraumatic meningitis with concealed CSF otorrhea. Pediatr Neurosci 15: 85-87, 1989

7) Rogers NL, James HE, Daleo G, Harvey LA: The third dimension in pediatric neurosurgery. A preliminary report. Childs Nerv Syst 9: 93-95, 1993

8) Tomura $N$, Miyauchi $T$, Shindo $M$, Seino $Y$, Watanabe M, Miura H, Watarai J, Kato T, Togawa K, Kowada M: Three-dimensional computed tomography in the head and neck diseases with bony abnormalities. Comput Med Imaging Graph 17: 411420, 1993

9) Unger JM, Gentry LR, Grossman JE: Sphenoid fractures: Prevalence, sites, and significance. Radiology 175: $175-180,1990$

Address reprint requests to: K. Yano, M.D., Department of Neurosurgery, Gifu Municipal Hospital, 7-1 Kashima-cho, Gifu 500, Japan. 